\title{
Editorial
}

\section{Fault Detection, Isolation, Estimation, and Accommodation of Dynamic Systems}

\author{
Ke Zhang, ${ }^{1}$ Peng Shi, ${ }^{2,3}$ Vincent Cocquempot, ${ }^{4}$ and Bin Jiang ${ }^{1}$ \\ ${ }^{1}$ College of Automation Engineering, Nanjing University of Aeronautics and Astronautics, 29 Yudao Street, Nanjing 210016, China \\ ${ }^{2}$ College of Automation, Harbin Engineering University, Harbin, Heilongjiang 150001, China \\ ${ }^{3}$ College of Engineering and Science, Victoria University, Melbourne, VIC 8001, Australia \\ ${ }^{4}$ CRIStAL Laboratory, UMR CNRS 9189, Lille 1 University of Sciences and Technologies, 59655 Villeneuve-d’Ascq Cedex, France
}

Correspondence should be addressed to Ke Zhang; kezhang@nuaa.edu.cn

Received 11 March 2015; Accepted 11 March 2015

Copyright $\odot 2015$ Ke Zhang et al. This is an open access article distributed under the Creative Commons Attribution License, which permits unrestricted use, distribution, and reproduction in any medium, provided the original work is properly cited.

Fault detection, isolation, estimation, and accommodation of dynamic systems play important roles to improve system reliability and stability. Hence, it is important to develop novel theories and methods to investigate fault diagnosis and fault tolerant control or related topics. The papers selected for this special issue represent a good panel for addressing this challenge. Of course, the selected topic and the papers are not an exhaustive representation of the area of fault detection, isolation, estimation, and accommodation of dynamic systems. Nonetheless, they represent the rich and many-facet knowledge, which we have the pleasure of sharing with the readers.

The special issue contains eleven papers, where one paper is about fault detection of discrete-time systems, two papers are related to the observer-based fault estimation, and two papers are regarding fault tolerant control of dynamical systems. Finally, six papers introduce the application of fault detection and fault tolerant control in practical systems.

In a paper entitled "Simultaneous Fault Detection and Control for Discrete-Time Systems via a Switched Scheme," J. $\mathrm{Li}$ and X. Dai study the problem of simultaneous fault detection and control for linear systems with a switched scheme. The switched detector/controller is designed simultaneously. When the faults are detected, the detector/controller is switched to reduce the effect of the faults.

In a paper entitled "Robust Fault Reconstruction in Discrete-Time Lipschitz Nonlinear Systems via EulerApproximate Proportional Integral Observers," Q. Jia et al. investigate the problem of observer-based robust fault reconstruction for a class of nonlinear sampled-data systems. A discrete-time Lipschitz nonlinear system is first established, and its Euler-approximate model is described; then, an Eulerapproximate proportional integral observer is constructed. Finally, a single-link flexible robot is employed to verify the effectiveness of the proposed fault-reconstructing method.

In a paper entitled "Robust Fault Diagnosis Design for Linear Multiagent Systems with Incipient Faults," J. Xia et al. investigate the design of a robust fault estimation observer for linear multiagent systems subject to incipient faults. By considering the fact that incipient faults are in low frequency domain, the fault estimation of such faults is proposed for discrete-time multiagent systems based on finite frequency technique.

In a paper entitled "Sensor Fault Tolerant Control of a Fast Steering Mirror System Using Adaptive PI-Based Sliding Mode Observer and Hardware Redundancy," H. Wang et al. consider a sensor fault tolerant control scheme for a two-axis fast steering mirror system with minimum power consumption and without changing the controller structure. An adaptive PI-based sliding mode observer is adopted to estimate the fault signal. The estimation is then used by the fault isolation logic to identify the fault.

In a paper entitled "Fault Tolerant Control for Uncertain Time-Delay Systems with a Trajectory Tracking Approach," S. Zhao et al. investigate the problem of fault tolerant control by trajectory tracking for a class of linear time-delay systems. 
Based on Lyapunov-Krasovskii functional approach, stability problems of the descriptor system are easily solved in terms of the linear matrix inequalities.

In a paper entitled "Bayesian Network Based Fault Prognosis via Bond Graph Modeling of High-Speed Railway Traction Device," Y. Wu et al. first introduce a bond graph modeling of CRH5 high-speed train traction device, based on which a multilayer Bayesian network is developed. Later, two different parameter learning algorithms such as Bayesian estimation and expectation maximization algorithm are adopted to determine the conditional probability table of the Bayesian network. Finally, Pearl's polytree propagation algorithm for joint probability reasoning is used for failure prediction.

In a paper entitled "Actuator Fault Diagnosis with Application to a Diesel Engine Testbed," B. Boulkroune et al. study the issues of actuator fault detection and isolation for diesel engines. They are particularly interested in faults affecting the exhaust gas recirculation and the variable geometry turbocharger actuator valves. A bank of observer-based residuals is designed using a nonlinear mean value model of diesel engines.

In a paper entitled "Joint Feature and Model Selection for SVM Fault Diagnosis in Solid Oxide Fuel Cell Systems," G. Moser et al. investigate an original technique for the joint feature and model selection in the context of support vector machine classification applied as a diagnosis strategy in model-based fault detection and isolation.

In a paper entitled "Vacuum Large Current Parallel Transfer Numerical Analysis," E. Dong et al. analyze the transfer current's experiments and the real vacuum arc resistance and precise correctional model in the large transfer current's process are obtained.

In a paper entitled "Relationship-Oriented Software Defined AS-Level Fast Rerouting for Multiple Link Failures," C. Li et al. study a local fast reroute approach to effectively recover from multiple link failures in one administrative domain. It can handle various types of multiple link failures that distribute in a vast scope of space and time.

In a paper entitled "SINS/CNS Nonlinear Integrated Navigation Algorithm for Hypersonic Vehicle," Y. Yu et al. design the model of altitude azimuth, platform error angles, and horizontal position. The SINS/CNS tightly integrated algorithm. Gaussian particle filter is introduced to solve the problem of nonlinear filtering.

\section{Acknowledgments}

We would like to thank the authors for their excellent contributions and patience in assisting us. Finally, the fundamental work of all the reviewers of these papers is also very warmly acknowledged.

$$
\begin{array}{r}
\text { Ke Zhang } \\
\text { Peng Shi } \\
\text { Vincent Cocquempot } \\
\text { Bin Jiang }
\end{array}
$$




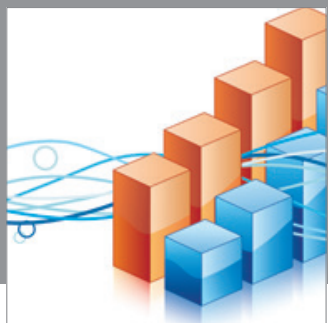

Advances in

Operations Research

mansans

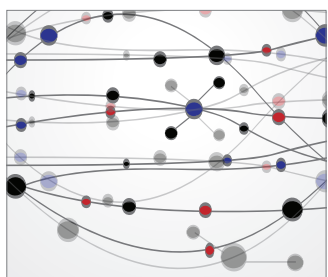

The Scientific World Journal
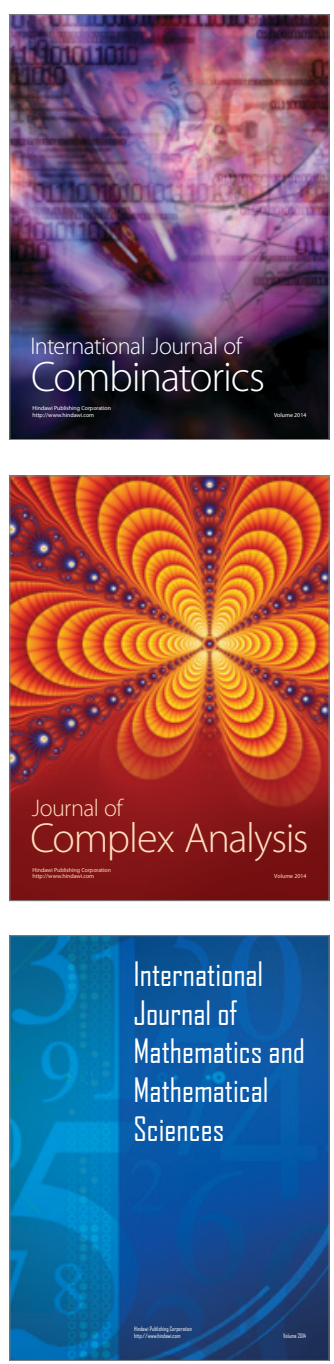
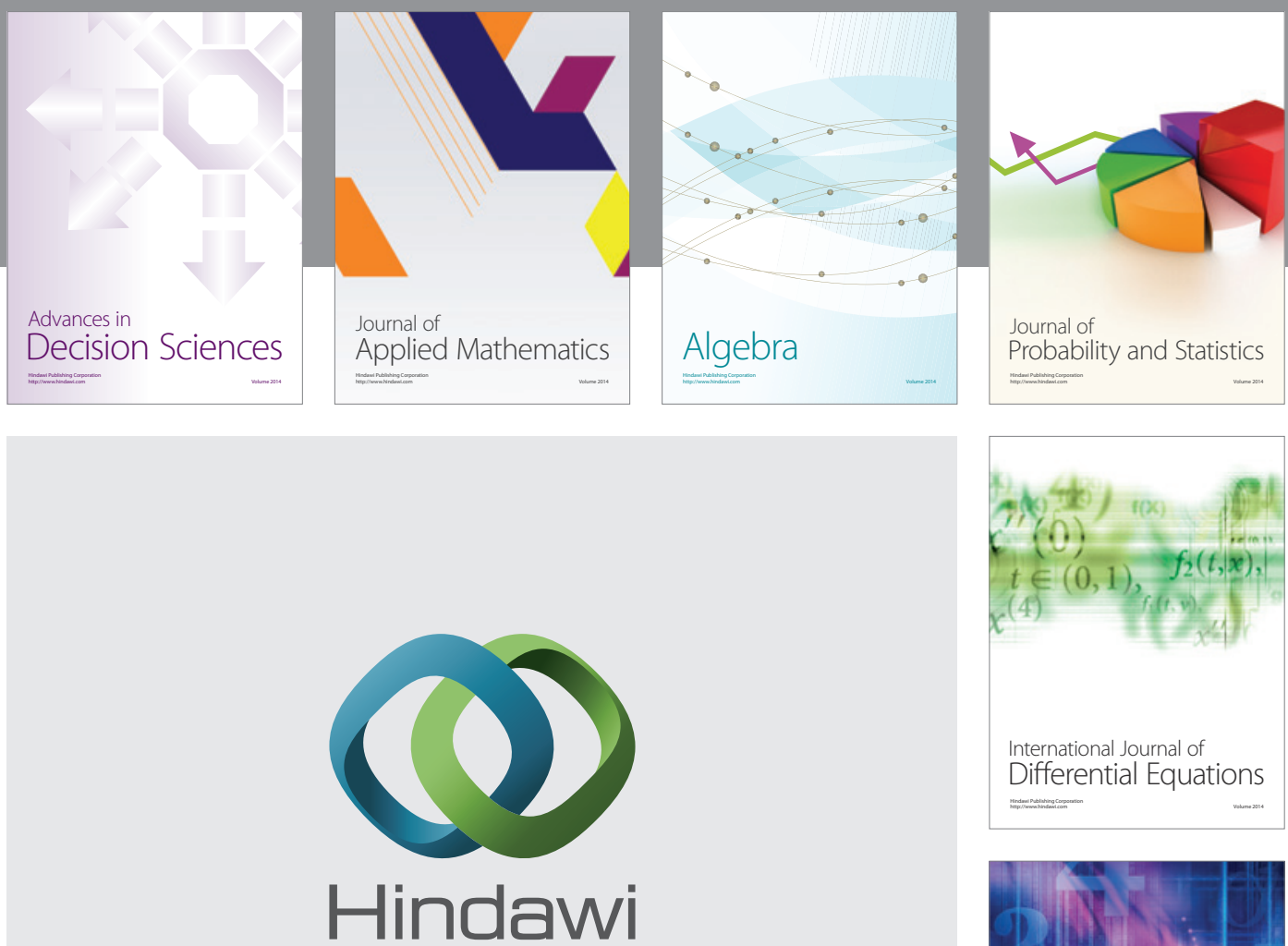

Submit your manuscripts at http://www.hindawi.com
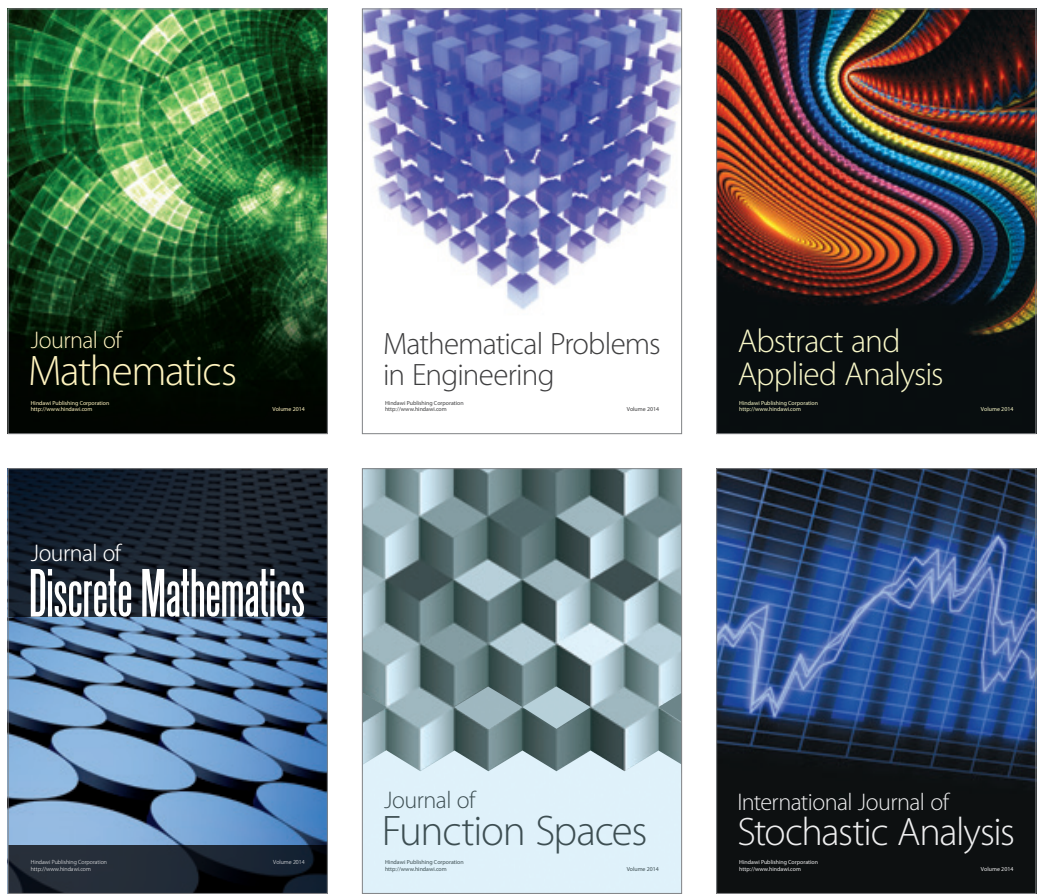

Journal of

Function Spaces

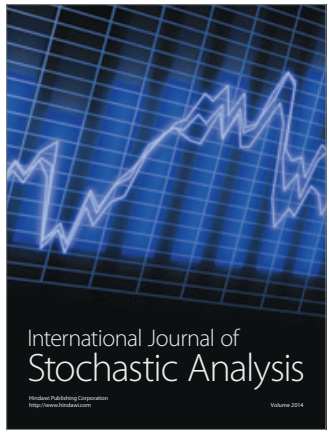

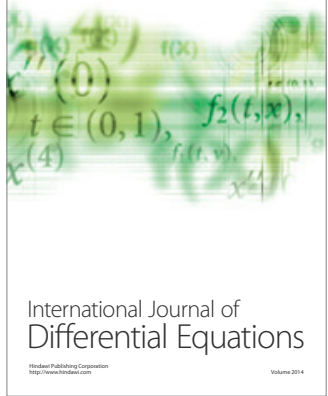
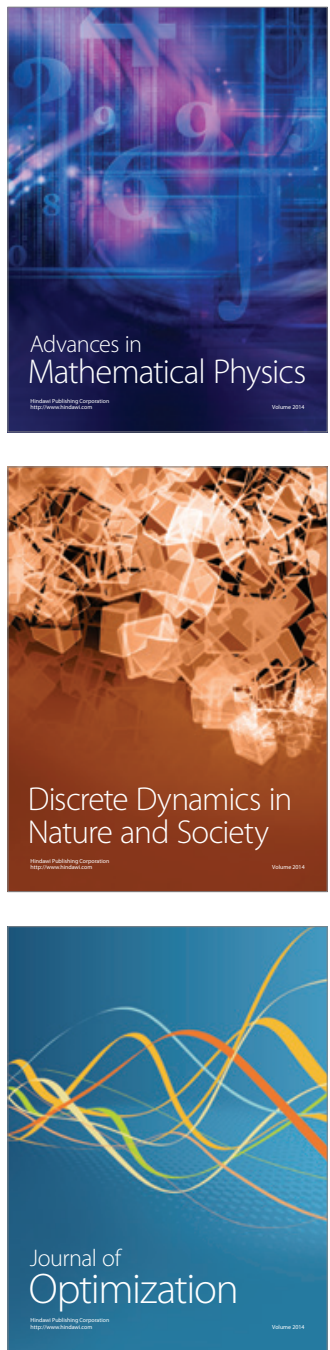